\title{
Muonium-antimuonium conversion
}

\author{
Lorenz Willmann ${ }^{\star}$ and Klaus Jungmann
}

Van Swinderen Institute, University of Groningen, 9747 AA, Groningen, The Netherlands

$\star$ L.Willmann@rug.nl

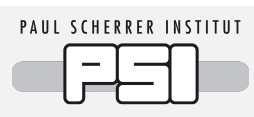

Review of Particle Physics at PSI

doi:10.21468/SciPostPhysProc.5

\begin{abstract}
The MACS experiment performed at PSI in the 1990s provided an yet unchallenged upper bound on the probability for a spontaneous conversion of the muonium atom, $\mathrm{M}=\left(\mu^{+} \mathrm{e}^{-}\right)$, into its antiatom, antimuonium $\overline{\mathrm{M}}=\left(\mu^{-} \mathrm{e}^{+}\right)$. It comprises the culmination of a series of measurements at various accelerator laboratories worldwide. The experimental limits on the process have provided input and steering for the further development of a variety of theoretical models beyond the standard theory, in particular for models which address lepton number violating processes and matter-antimatter oscillations. Several models beyond the standard theory could be strongly disfavored. There is interest in a new measurement and improved sensitivity could be reached by exploiting the time evolution of the conversion process, e.g., at intense pulsed muonium sources.
\end{abstract}

(c) () Copyright L. Willmann and K. Jungmann. This work is licensed under the Creative Commons Attribution 4.0 International License. Published by the SciPost Foundation.
Received 16-02-2021

Accepted 28-04-2021

Published 06-09-2021

doi:10.21468/SciPostPhysProc.5.009

\subsection{Introduction}

The bound state of a positive muon $\left(\mu^{+}\right)$and an electron $\left(\mathrm{e}^{-}\right)$is an exotic atom which has been named muonium (M) by V. Telegdi. This exotic atom was first produced and observed by V.W. Hughes and collaborators in 1960 [1]. It is well suited for precision experiments as it consists of two point-like leptons of different masses that belong to two different particle generations. The constituents of the $\mathrm{M}$ atom experience a rather long interaction time, which ultimately is limited by the muon lifetime $\tau_{\mu}=2.2 \mu s$ [2]. The $\mathrm{M}$ atom has been employed for series of precision measurements. The results can be used to make precise tests of theory, in particular Quantum Electrodynamics. Due to the absence of direct strong interactions between the two constituents, the properties of M can be calculated within the Standard Model (SM) to very high accuracy. Precise experiments yield accurate values of different fundamental constants such as the muon mass $\mathrm{m}_{\mu}$ and the electromagnetic fine structure constant $\alpha$. Further, tests of fundamental symmetries, among which are lepton universality and the equality of the muon and electron electric charges, $\mathrm{q}_{\mathrm{e}} / \mathrm{q}_{\mu}$, can be conducted, and scrutiny of lepton family number conservation is enabled [3]. 


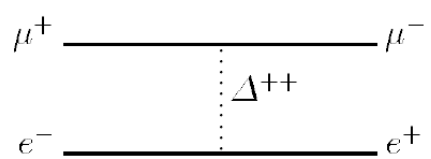

(a)

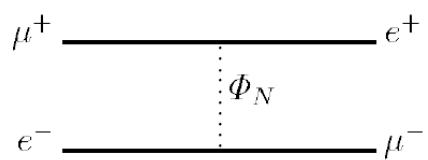

(c)
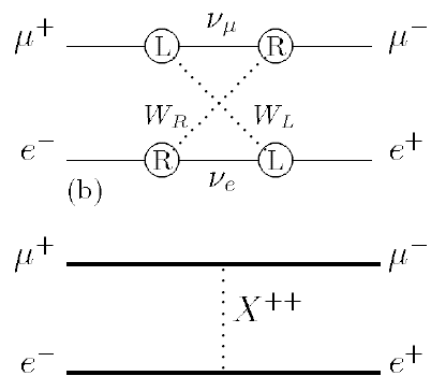

(d)

Figure 9.1: $\quad \mathrm{M}-\overline{\mathrm{M}}$ conversion for various scenarios beyond the Standard Model. (a) Doubly charged Higgs bosons $\Delta^{++}$, (b) heavy Majorana neutrinos, (c) neutral scalars $\Phi_{N}$, or(d) a bileptonic gauge boson $\mathrm{X}^{++}$could mediate the process (from [4]).

Spontaneous conversion of muonium $\mathrm{M}$ into antimuonium $\overline{\mathrm{M}}$ would violate additive lepton family (generation) number conservation by two units. Like other processes such as $\mu \rightarrow \mathrm{e} \gamma$, $\mu \rightarrow$ eee, $\mu+\mathrm{Z} \rightarrow \mathrm{e}+\mathrm{Z}$ and the decay mode $\mu^{+} \rightarrow \mathrm{e}^{+}+v_{\mu}+\overline{v_{e}}[2]$, M- $\overline{\mathrm{M}}$ conversion is not allowed in the Standard Model. Charged leptons appear to observe lepton family number. There is no guidance from theory as to which of these various rare decay modes beyond the SM would be more favored by nature. Therefore searches for all of them are well motivated. A series of experiments searching for $\mathrm{M}-\overline{\mathrm{M}}$ conversion with ever increasing sensitivity was started in the mid 1960s. They yielded various strong limits on speculative theories [4], such as leftright symmetry, supersymmetry, 3-1-1 models and others (Figure 9.1). Numerous theoretical models have been proposed over the past decades [5-8], where lepton family number violation is a natural feature and where $\mathrm{M}-\overline{\mathrm{M}}$ conversion is an essential part.

Oscillations in the lepton sector between neutrinos of different flavors have been observed and are the subject of ongoing precision experiments [9] in a very active field. $\mathrm{K}^{0}-\overline{\mathrm{K}^{0}}$ and $\mathrm{B}^{0}-\overline{\mathrm{B}^{0}}$ oscillations are well established in the quark sector [2]. The $\mathrm{K}^{0}$ particle consists of two quarks from the 1st and the 2nd quark generations, i.e., it is the quark analogue of $\mathrm{M}$, which consists of charged leptons from the 1st and 2nd lepton generations. Non-observation of spontaneous conversion of $\mathrm{M}$ into $\overline{\mathrm{M}}$ (or even oscillations between particle and antiparticle) makes it an intriguing puzzle waiting for explanation.

Historically the $\mathrm{M}-\overline{\mathrm{M}}$ conversion process has been described via effective four fermion interaction with a coupling constant $\mathrm{G}_{\mathrm{M}} \overline{\mathrm{M}}$, which can be compared to the Fermi coupling constant $\mathrm{G}_{\mathrm{F}}$ in weak interactions [10]. For a system starting as an $\mathrm{M}$ atom at time $t=0$, we have at a later time $t$ the probability

$$
\mathrm{P}_{\mathrm{MM}}(t)=\left(\frac{\delta t}{2 \hbar}\right)^{2} \cdot \exp \left(-\frac{t}{\tau_{\mu}}\right)
$$

to observe it as $\overline{\mathrm{M}}$, where

$$
\delta=\frac{8 \mathrm{G}_{\mathrm{F}}}{\sqrt{2} \mathrm{n}^{2} \pi \mathrm{a}_{0}^{3}} \frac{\mathrm{G}_{\mathrm{MM}}}{\mathrm{G}_{\mathrm{F}}},
$$

with $\mathrm{a}_{0}$ the $\mathrm{M}$ Bohr radius and $\mathrm{n}$ the atomic state principal quantum number. Integrating (9.1) over all times yields

$$
\mathrm{P}_{\mathrm{MM}}=2.56 \cdot 10^{-5} \frac{\mathrm{G}_{\mathrm{MM}}}{\mathrm{G}_{\mathrm{F}}} \text {. }
$$


In external magnetic fields the degeneracy of energy levels in $\mathrm{M}$ and $\overline{\mathrm{M}}$ is lifted and hence the conversion probability $\mathrm{P}_{\mathrm{M}}$ is reduced $[11,12]$. At a magnetic field strength of $1 \mathrm{kG}$ the probability is reduced to $\approx 35 \%$ its value at $0 \mathrm{kG}$.

Collisions of $\mathrm{M}$ atoms in gases or condensed matter lead to further substantial suppression of $\mathrm{P}_{\mathrm{M}}$, which can be orders of magnitude depending on the material density. The first search for $\mathrm{M}-\overline{\mathrm{M}}$ conversion at the NEVIS cyclotron was performed in $1 \mathrm{~atm} \mathrm{Ar}$ gas, where $\mathrm{M}$ can be produced efficiently. Thus the experiment established a rather high limit of $\mathrm{G}_{\mathrm{MM}}<5800 \mathrm{G}_{\mathrm{F}}$ [13]. Substantial progress was made after the discovery that $\mathrm{M}$ produced inside $\mathrm{SiO}_{2}$ powder grains can emerge into a surrounding vacuum [14]. This discovery started a number of new and successful experiments (for more details see e.g. [15]).

\subsection{The PSI M- $\overline{\mathrm{M}}$ Experiment}

The latest and most precise experiment was conducted with MACS, the Muonium Antimuonium Conversion Spectrometer at PSI. Data were taken at the PSI beamlines $\pi E 3$ and $\pi E 5$ [4]. In the course of $1730 \mathrm{~h}$ data taking $\mathrm{M}$ atoms were produced in $\mathrm{SiO}_{2}$ powder target from which they emerged with an efficiency of several per cent of the stopped muons into vacuum. A $\mu^{+}$ beam momentum of order $21 \mathrm{MeV} / \mathrm{c}$ and a very narrow momentum bite of order $1 \%$, was essential for this rather high yield so that the $\mu^{+}$could be efficiently stopped near the surface of a fluffy $\mathrm{SiO}_{2}$ powder target. In total the decay of $5.6(2) \cdot 10^{10} \mathrm{M}$ atoms in vacuo were monitored. This permitted the establishment of a limit on the probability for $\mathrm{M}-\overline{\mathrm{M}}$ conversion of $\mathrm{P}_{\mathrm{MM}} \leq 8.3(3) \cdot 10^{-11}$ (90\% C.L.). This is a substantial improvement over previous other projects [2].

The MACS (Figure 9.2) design manifests the strong symmetry in the detection signatures for $\mathrm{M}$ and $\overline{\mathrm{M}}$. The signature used for constant monitoring of $\mathrm{M}$ production rates provided for crucial calibration information of all parts of the detector with good accuracy. Monitoring the $\mathrm{M}$ yield every $\approx 5 \mathrm{~h}$ for $\approx 15$ min proved indispensable as the $\mathrm{SiO}_{2}$ targets deteriorated within a week. Targets were replaced once the yield had dropped by 50\%. MACS has an acceptance of $0.71 \mathrm{sr}$ for the detection of the Michel $\mathrm{e}^{+} / \mathrm{e}^{-}$and $4 \pi$ extraction of the atomic shell $\mathrm{e}^{-} / \mathrm{e}^{+}$. The high energy decay $\mathrm{e}^{-} / \mathrm{e}^{+}$are detected in the cylindrical magnetic spectrometer (SINDRUM I) operated at $\mathrm{B}=1 \mathrm{kG}$ magnetic field. The magnetic spectrometer consisted of 5 proportional wire chambers equipped with cathode strip readout and a plastic scintillator hodoscope for timing purposes. SINDRUM I had been refurbished with a new electronic hardware pipeline system for the wire chambers which had $100 \mathrm{MHz}$ clock rate and 256 cycle pipeline depth. The $\mathrm{e}^{+} / \mathrm{e}^{-}$from $\mu^{+} / \mu^{-}$-decays have a continuous energy (Michel) spectrum with energies up to $\mathrm{E}=1 / 2 \cdot \mathrm{m}_{\mu} \cdot \mathrm{c}^{2}=53 \mathrm{MeV}$. The momentum resolution for positrons at the highest energy has been determined to be 54(2)\% in the spectrometer. This value was dominated by the $2 \mathrm{~mm}$ spacing between wires in the cylindrical wire chambers.

$\mathrm{M}(\overline{\mathrm{M}})$ atom decays were identified through a coincidence signature between high energy $\mathrm{e}^{+}\left(\mathrm{e}^{-}\right)$from muon decay in the magnetic spectrometer, and the low energy atomic shell $\mathrm{e}^{-}$ $\left(\mathrm{e}^{+}\right)$which was transported and detected at the MCP/CsI detector. The low energy particles had average kinetic energies equaling the $M(\bar{M})$ atomic binding energy $E_{b}=13.6 \mathrm{eV}$. The intrinsic 16(2)\% efficiency of the MCP for $10 \mathrm{keV} \mathrm{e}-\left(\mathrm{e}^{+}\right)$was enhanced 4-fold by a MgO coated $\mathrm{C}$ foil a few $\mathrm{mm}$ in front of it [16]. The pipeline readout system enabled an efficient readout after a trigger from the full coincidence. This resulted in a readout rate in $\overline{\mathrm{M}}$ search mode of order a few s ${ }^{-1}$ for muon beam intensities of order $10^{7} \mathrm{~s}^{-1}$. The clean coincidence signature resulted in the suppression of the accidental combinatoric background to about 1 for the total collected statistics. The main limitation for further data collection arises from allowed physical processes. These are presented in Figure 9.3 which display sample time-offlight (TOF) spectra of possible background as it arises from Bhabha scattering and the low energy tail of the decay $\mu \rightarrow 3 e 2 v$. 


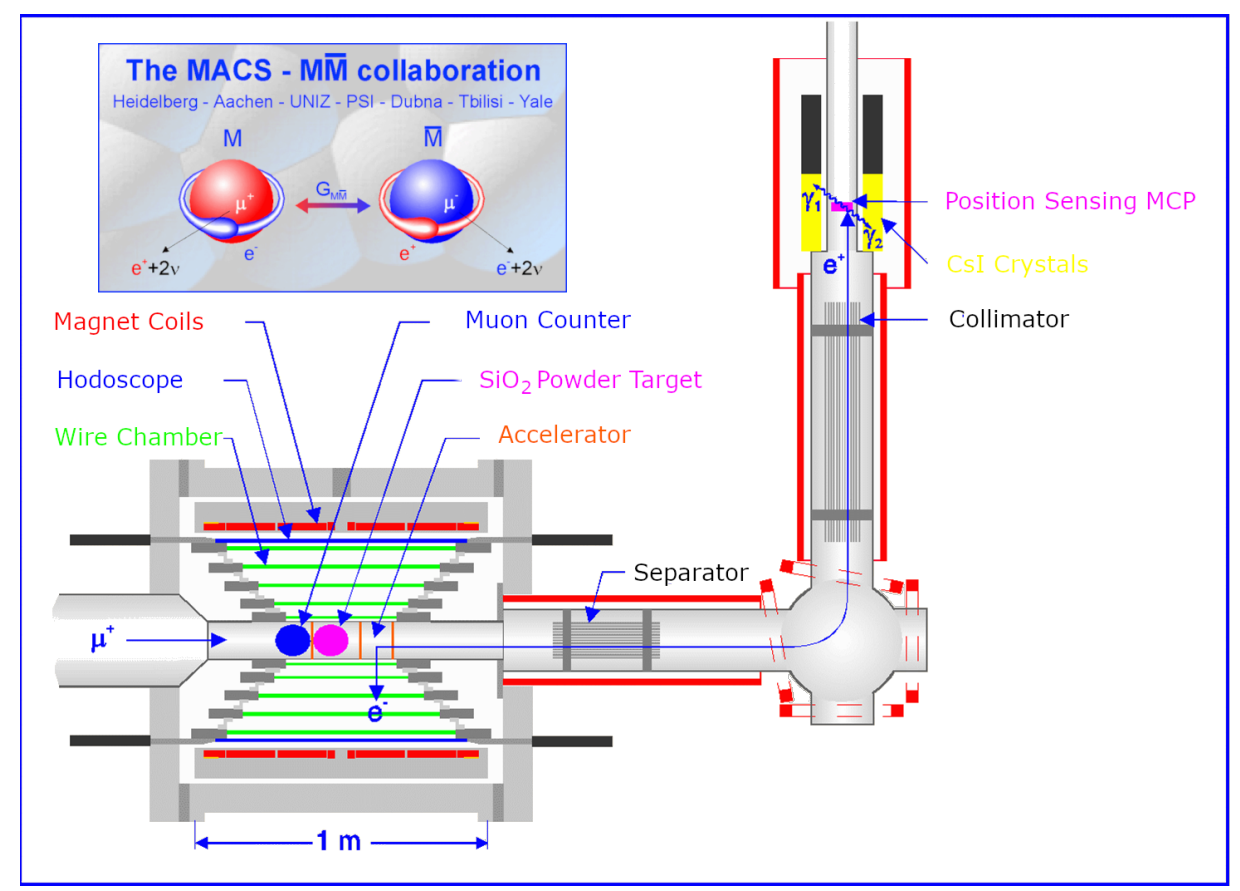

Figure 9.2: The MACS setup consists of the refurbished SINDRUM I magnetic spectrometer for detection of Michel $\mathrm{e}^{+} / \mathrm{e}^{-}$from $\mu^{+} / \mu^{-}$-decay combined with a transport and imaging system for atomic shell $\mathrm{e}^{-} / \mathrm{e}^{+}$. The detector comprises maximum symmetry for the detection of $\mathrm{M}$ and $\overline{\mathrm{M}}$. Switching between M-mode for monitoring M-production and $\overline{\mathrm{M}}$-search-mode was achieved by reversing the magnetic field directions and changing the $10 \mathrm{keV}$ extraction voltage polarity for the atomic shell particle remaining after $\mathrm{M} / \overline{\mathrm{M}}$-decay. The spectrometer consists of five cylindrical wire chambers and a hodoscope for timing. The axial magnetic field in the transport system provided for axial confinement and retracing of the position information from a microchannel plate (MCP) detector to obtain the decay vertex with $8.0(4) \mathrm{mm}$ resolution. Further background suppression in $\overline{\mathrm{M}}$-mode is provided by an electrostatic separator and a collimator in the transport system as well as $\mathrm{e}^{+}$identification via annihilation $\gamma \mathrm{s}$ in CsI crystals near the MCP.

The M- $\overline{\mathrm{M}}$ experiment collected data in three stages. Between these stages several substantial upgrades were implemented. In particular using a cathode strip readout of the wire chambers proved essential since it improved the 3D reconstruction of the vertex between the Michel particle and the low energy atomic shell particle detected on the position sensitive MCP detector. Data were recorded for a total of $1730 \mathrm{~h}$ in the overall experiment. One candidate event survived the analysis with stringent cuts on the reconstructed vertex, TOF and required $511 \mathrm{keV} \gamma$-detection for positron identification (Figure 9.4). The resulting limit on $\mathrm{P}_{\mathrm{MM}}$ corresponds to an upper limit on the coupling constant in an effective 4 fermion coupling of $\mathrm{G}_{\mathrm{M}} \overline{\mathrm{M}}<3.0 \cdot 10^{-3} \mathrm{G}_{\mathrm{F}}$. The experiment was limited in its sensitivity by physical background in the acceptance of the detector.

\subsection{Conclusions}

$\mathrm{M}-\overline{\mathrm{M}}$ conversion is of great interest and new experiments with improved apparatus exploiting the time dependence of the conversion process could reach substantially more stringent bounds [15]. In the recent years the upper limit established in the MACS experiment has been 

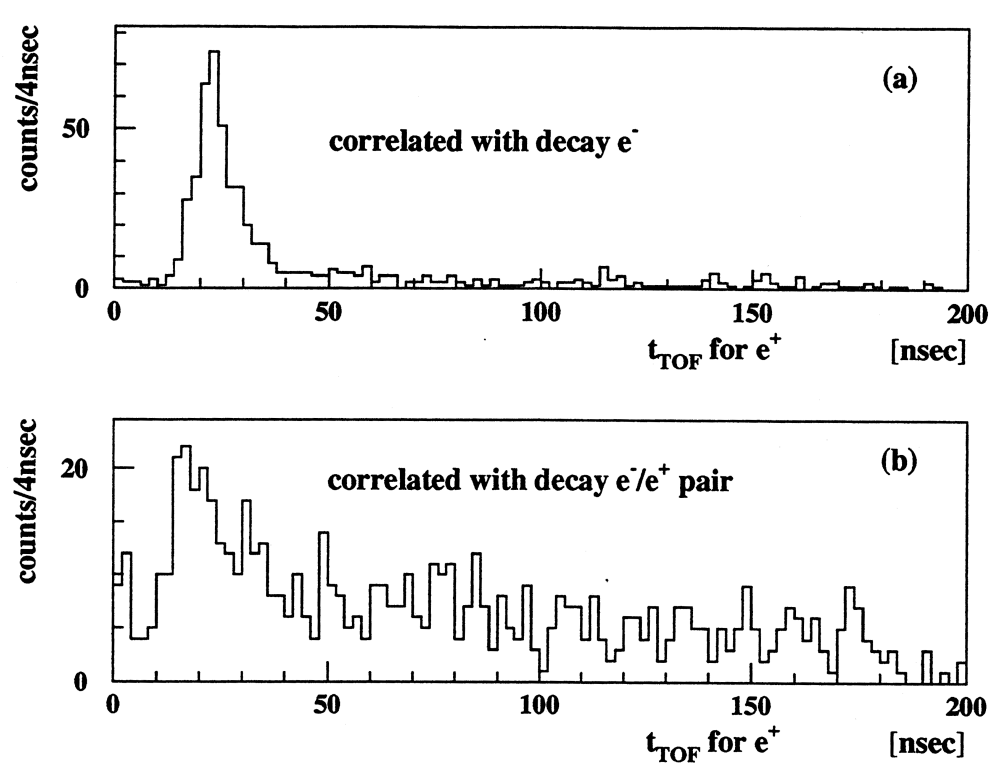

Figure 9.3: Dominant physical background observed in $440 \mathrm{~h}$ of running when relaxing the stringent coincidence requirements. (a) Bhabha scattering of Michel $\mathrm{e}^{+}$ electrons in the support structure. (b) A small fraction of phase space for allowed $\mu \rightarrow 3 e 2 v$ decay results in $\mathrm{e}^{+} / \mathrm{e}^{-}$pairs detected by the magnetic spectrometer coincident with a low energy $\mathrm{e}^{+}$within the acceptance of the detector. The expected arrival time for a $\mathrm{e}^{+}$from $\overline{\mathrm{M}}$-decay is 78.1(1)ns.

exploited to disfavor single flavor-violating axion-like particle (ALP) based explanations for anomalies observed in electron and muon g-2 measurements [6]. Improved future $\mathrm{M}-\overline{\mathrm{M}}$ experiments can probe a similar parameter space as experiments at a future lepton collider which are searching for charged lepton flavor violation via, e.g., on-shell production of bileptons [7]. In view of this a new $\mathrm{M}-\overline{\mathrm{M}}$ would be very well motivated.

Since the MACS experiment reached its possible sensitivity limit, an improved concept and a refined setup are required to establish tighter bounds. At a pulsed muon source one can benefit from exploiting the time evolution of the conversion process [15]. All muon decay related background decreases on a time scale given by the $\mu^{+}$lifetime. For an $\mathrm{n}$-fold coincidence signature this background drops significantly with $\exp \left(-\mathrm{n} \cdot \frac{t}{\tau_{\mu}}\right)$. The probability of finding $\overline{\mathrm{M}}$ grows in time to a maximum at $2 \tau_{\mu}$ (see Figure 9.5). Thus the ratio of $\mathrm{M}$ to $\overline{\mathrm{M}}$ decays grows with $t^{2}$. In case of a multiple coincidence, as in MACS, this implies that the potential $\overline{\mathrm{M}}$ signal/background increased. Therefore a new experiment should be considered, e.g., in connection with the muon source of a muon collider, provided high muon beam quality, i.e. a narrow $\mu^{+}$momentum band at subsurface $\mu^{+}$momentum. We note that for such an improved experiment beam repetition rates of up to several $10 \mathrm{kHz}$ with $\mu^{+}$bunches of up to $\approx \mu$ s length would be ideal.

With a new experiment, from the viewpoint of signal to background ratio, an improved value for $G_{M \bar{M}}$ by at least 2 orders of magnitude should be possible, i.e., 4 orders of magnitude in the conversion probability. At such sensitivity there would be strong constraints for the development of models beyond standard theory [5-8]. 

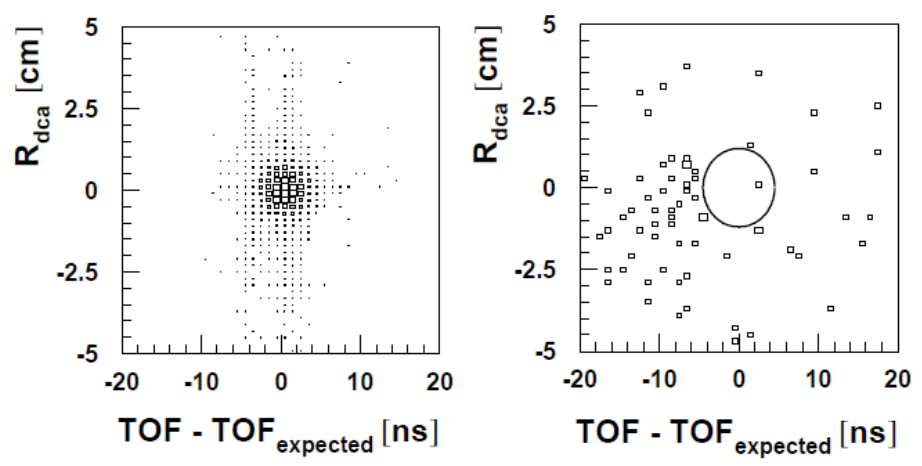

Figure 9.4: The distribution of the distance of closest approach $\mathrm{R}_{\text {dcad }}$ between a trace from a particle registered in the magnetic spectrometer and the back-projected position on the MCP as a function of the TOF for the atomic shell particle as measured for $\mathrm{M}$ atoms (left). The data recorded in the final data-taking period of $1290 \mathrm{~h}$ searching for $\overline{\mathrm{M}}$ (right).

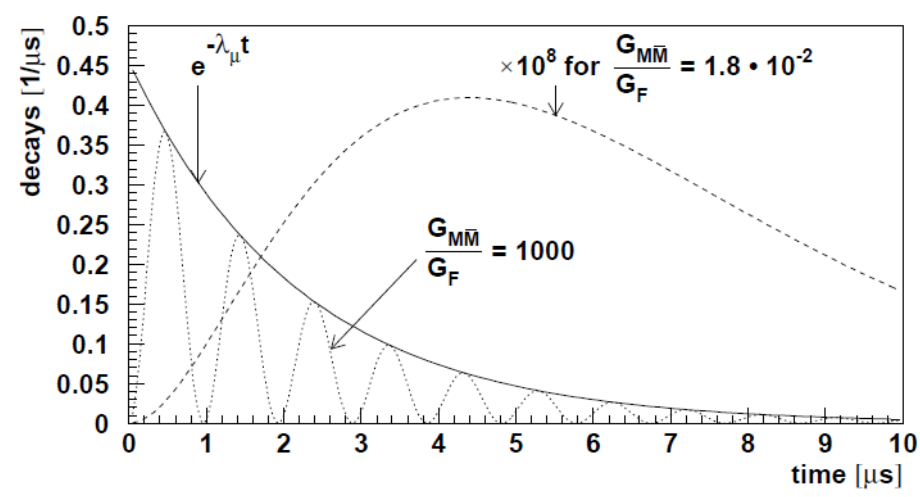

Figure 9.5: The probability for observing an $\bar{M}$ decay increases with time and reaches a maximum at about $2 \tau_{\mu}$. In particular he ratio of $\bar{M}$ to $\mu^{+}$-decays increases further with time. Therefore an enhanced signal to background ratio could be expected from experiments in which the time from $\mathrm{M}$ formation and the subsequent $\mathrm{M}$ - or $\overline{\mathrm{M}}$-decay can recorded [15]. This would favor future experiments at intense future pulsed muon sources [17].

\section{Acknowledgments}

The authors are indebted to the MACS collaboration which conducted the latest experiment in the last decade of the past millennium. Setting up and testing occurred in the early 1990's and the final data collection runs were in 1995-96. We have experienced very strong commitment through hardware contributions, conceptual input and proficiency in data analysis from all international collaborators in Aachen, Dubna, Heidelberg, Tiblisi, Yale and Zürich. Through this the completion of the experiment in rather short time was possible. We like to dedicate this article to our local co-spokesperson, the late Willi Bertl of PSI. 


\section{References}

[1] M. E. Zeller, ed., A Festschrift in honor of Vernon W. Hughes, Proceedings, Symposium, New Haven, USA (1992).

[2] M. Tanabashi et al., Review of particle physics, Phys. Rev. D 98, 030001 (2018), doi:10.1103/PhysRevD.98.030001.

[3] K. P. Jungmann, Precision muonium spectroscopy, J. Phys. Soc. Jpn. 85, 091004 (2016), doi:10.7566/JPSJ.85.091004.

[4] L. Willmann et al., New bounds from a search for muonium to antimuonium conversion, Phys. Rev. Lett. 82, 49 (1999), doi:10.1103/PhysRevLett.82.49.

[5] A. Ilakovac, Lepton flavor violation in the standard model extended by heavy singlet dirac neutrinos, Phys. Rev. D 62, 036010 (2000), doi:10.1103/PhysRevD.62.036010.

[6] M. Endo, S. Iguro and T. Kitahara, Probing e $\mu$ flavor-violating ALP at Belle II, J. High Energy Phys. 06, 040 (2020), doi:10.1007/JHEP06(2020)040.

[7] T. Li and M. A. Schmidt, Sensitivity of future lepton colliders and low-energy experiments to charged lepton flavor violation from bileptons, Phys. Rev. D 100, 115007 (2019), doi:10.1103/PhysRevD.100.115007.

[8] P. Bhupal Dev and Y. Zhang, Displaced vertex signatures of doubly charged scalars in the type-II seesaw and its left-right extensions, J. High Energy Phys. 10, 199 (2018), doi:10.1007/JHEP10(2018)199.

[9] J. Bernabeu, Symmetries and their breaking in the fundamental laws of physics, SymmetryBasel 12, 1316 (2020), doi:10.3390/sym12081316.

[10] G. Feinberg and S. Weinberg, Conversion of muonium into antimuonium, Phys. Rev. 123, 1439 (1961), doi:10.1103/PhysRev.123.1439.

[11] K. Horikawa and K. Sasaki, Muonium-antimuonium conversion in models with dilepton gauge bosons, Phys. Rev. D 53, 560 (1996), doi:10.1103/PhysRevD.53.560.

[12] W.-S. Hou and G.-G. Wong, Muonium-antimuonium transitions via neutral scalar bosons, Phys. Rev. D 53, 1537 (1996), doi:10.1103/PhysRevD.53.1537.

[13] J. Amato, P. Crane, V. Hughes, J. Rothberg and P. Thompson, Search for muonium-antimuonium conversion, Phys. Rev. Lett. 21, 1709 (1968), doi:10.1103/PhysRevLett.21.1709.

[14] G. Marshall, J. Warren, D. Garner, G. Clark, J. Brewer and D. Fleming, Production of thermal muonium in vacuum between grains of fine silica powders, Phys. Lett. A 65, 351 (1978), doi:10.1016/0375-9601(78)90727-2.

[15] L. Willmann and K. Jungmann, The muonium atom as a probe of physics beyond the standard model, Lect. Notes Phys. 499, 43 (1997), doi:10.1007/BFb0104314.

[16] P. Schmidt et al., An efficient position sensitive detector for 3 to $30 \mathrm{kev}$ positrons and electrons, Nucl. Instr. Meth. Phys. Res. Sec. A 376, 139 (1996), doi:https://doi.org/10.1016/0168-9002(96)00267-7.

[17] J. Äystö et al., Physics with low-energy muons at a neutrino factory complex (2001), arXiv:hep-ph/0109217. 